\title{
A Probabilistic Approach to Delineating Functional Brain Regions
}

\author{
Jan Kalbitzer ${ }^{1}$, Claus Svarer ${ }^{1}$, Vibe G. Frokjaer ${ }^{1}$, David Erritzoe ${ }^{1}$, William F.C. Baaré2 ${ }^{\text {, Jacob Madsen }}{ }^{3}$, \\ Steen G. Hasselbalch ${ }^{1}$, and Gitte M. Knudsen ${ }^{1}$ \\ ${ }^{I}$ Neurobiology Research Unit, Copenhagen University Hospital, Copenhagen, Denmark; ${ }^{2}$ Danish Research Center for Magnetic \\ Resonance, Copenhagen University Hospital, Hvidovre, Denmark; and ${ }^{3}$ PET and Cyclotron Unit, Copenhagen University Hospital, \\ Copenhagen, Denmark
}

\begin{abstract}
The purpose of this study was to develop a reliable observerindependent approach to delineating volumes of interest (VOIs) for functional brain regions that are not identifiable on structural MR images. The case is made for the raphé nuclei, a collection of nuclei situated in the brain stem known to be densely packed with serotonin transporters (5-hydroxytryptaminic [5-HTT] system). Methods: A template set for the raphé nuclei, based on their high content of $5-\mathrm{HTT}$ as visualized in parametric ${ }^{11}$ C-labeled 3-amino-4-(2-dimethylaminomethyl-phenylsulfanyl)benzonitrile PET images, was created for 10 healthy subjects. The templates were subsequently included in the region sets used in a previously published automatic MRI-based approach to create an observer- and activity-independent probabilistic VOI map. The probabilistic map approach was tested in a different group of 10 subjects and compared with a manual delineation approach. Results: In addition to providing an observer-independent solution, the probabilistic map approach returned a higher specific binding determined in a larger region, ultimately providing better data fitting in kinetic modeling. Conclusion: We developed a fast, observer-independent, reliable approach to delineating regions that can be identified only by functional imaging, here exemplified by the raphé nuclei. This approach can be used in future studies to create functional VOI maps based on neuroreceptor fingerprints retrieved through in vivo brain imaging.
\end{abstract}

Key Words: raphé nuclei; volume of interest; serotonin transporter; DASB

J Nucl Med Technol 2009; 37:91-95

DOI: $10.2967 /$ jnmt.108.054056

$\mathbf{T}$ he raphé nuclei are located in the brain stem and constitute the core of the 5-hydroxytryptaminic (5-HTT) (serotonin) system (1). Alterations in general morphology and serotonin activity in this area have been reported in depression (2-5) and bipolar disorder (4,5) and also in

Received May 2, 2008; revision accepted Feb. 23, 2009.

For correspondence or reprints contact: Jan Kalbitzer, Neurobiology Research Unit, Copenhagen University Hospital, Blegdamsvej 9, 2100, Copenhagen, Denmark.

E-mail: jan@kalbitzer.net

COPYRIGHT $\odot 2009$ by the Society of Nuclear Medicine, Inc. degenerative diseases (6). Accordingly, the raphé nuclei constitute a highly interesting brain region for PET in neuropsychiatric research on the serotonin system. However, delineation of an area for the raphé nuclei is complicated by the anatomic location and structure of the region. The nuclei consist of a heterogeneous group of cells around the midline of the brain stem that is poorly defined toward the surrounding tissue; it is, therefore, difficult to identify not only structurally but also histologically the raphé nuclei in brain images (1).

So far, the raphé nuclei region has been defined manually, either directly on the functional image or on a coregistered MR image, by the placement of circles with a fixed diameter on consecutive slides directly on PET scans, around the center of highest activity $(2,7)$; placement of a circle of a fixed diameter on MR images in the midbrain in front of the aqueduct (5); or direct delineation on PET images, without further description $(8,9)$. These approaches are, however, prone to be biased by physiologic and pathologic activity alterations in this area and do not fully take into account the individual shape and size of the brain. Furthermore, these methods are highly user-dependent and are likely to be associated with high inter- and intraobserver variability.

Here, we describe a probability map-based automatic approach for the delineation of the raphé nuclei on MR and PET images. The method is strictly observer-independent and based on a probabilistic mapping algorithm to delineate anatomic regions, as described earlier (10). In 10 subjects, we identified the raphé region in the PET images and transferred the volume of interest (VOI) to the coregistered MR images; these 10 MRI templates were then subsequently used as templates when creating a probability-based VOI in 10 new subjects.

\section{MATERIALS AND METHODS}

\section{Subjects}

Twenty volunteers were recruited by newspaper advertisement. MR and PET images from 10 of the healthy subjects (age, 22-40 y; 5 women) were used to establish templates for the raphé nuclei region, and the template was subsequently applied to images from the other 10 healthy volunteers (age, 25-63 y; 6 women). The 
protocol was approved by the Ethics Committee of Copenhagen and Frederiksberg ((KF) 01-156/04, (KF) 01-124/04, and (KF) 11283038). Exclusion criteria included history of medical or neuropsychiatric disorders and drug abuse.

\section{PET and MRI Coregistration}

PET scans were obtained with an 18-ring scanner (Advance; GE Healthcare), operating in 3-dimensional acquisition mode and producing 35 image slices with an interslice distance of $4.25 \mathrm{~mm}$. The final PET voxel size was $2 \times 2 \times 4.25 \mathrm{~mm}$. After a 10 -min transmission scan, a dynamic 90-min emission scan was initiated on intravenous injection of ${ }^{11} \mathrm{C}$-labeled 3-amino-4-(2-dimethylaminomethyl-phenylsulfanyl)-benzonitrile ( ${ }^{11} \mathrm{C}$-DASB) (246$590 \mathrm{MBq}$ ) for $12 \mathrm{~s}$. The acquisition consisted of 36 time frames, increasing progressively in duration from $10 \mathrm{~s}$ to $10 \mathrm{~min}$. The attenuation- and decay-corrected recordings were reconstructed by filtered backprojection using a Hann filter (6 mm).

High-resolution 3-dimensional T1-weighted, sagittal, spoiled gradient echo scans of the head were acquired on a 3-T MRI scanner (Magnetom Trio; Siemens) with an 8-channel head coil (Invivo). The MRI voxel size was $1 \times 1 \times 1 \mathrm{~mm}$.

The emission recording across frames 10-36 was automatically aligned to frame 26. Then the PET image (using an average of frames 10-36) was coregistered to the MR image using the automated image registration algorithm (http://bishopw.loni.ucla.edu/ AIR5/); the quality of each coregistration was evaluated visually.

\section{Quantification and Region Assessment}

The radioligand ${ }^{11} \mathrm{C}$-DASB binds with high affinity and selectivity to 5-HTT sites (11). For quantification, we used the modified reference tissue model with $k_{2}^{\prime}$ calculated for each subject individually, as described by Ichise et al. (12).

PMOD (version 2.85; PMOD Technologies) was used for the kinetic modeling; this software returns a value, $\chi^{2}$, that describes the deviation between estimated and actual time-activity curves (i.e., goodness of fit) based on a cost function.

\section{Delineation of VOI}

First, parametric images of the specific binding $\left(\mathrm{BP}_{\mathrm{ND}}\right)$ of ${ }^{11} \mathrm{C}$ DASB were created. On ${ }^{11} \mathrm{C}$-DASB parametric images, the rostral raphé nuclei can easily be identified as the center of highest activity (Fig. 1). However, a delineation of the raphé region by the application of a fixed activity threshold directly on the PET image is prone to be biased by physiologic and pathologic alterations. Instead, we used an extension of the method described by Svarer et al. (10) in which the functional raphé nuclei region defined from binding potential images is included in MRI VOI template sets.

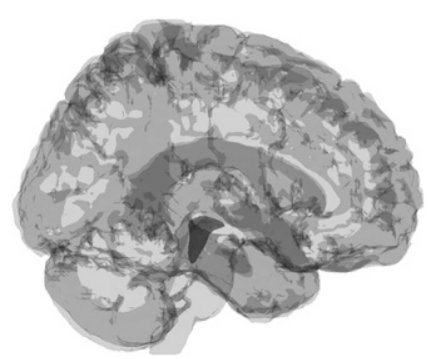

FIGURE 1. Three-dimensional overlay of MR image and parametric map of ${ }^{11} \mathrm{C}$-DASB PET image representing $\mathrm{BP}_{\mathrm{ND}}$ to $5-\mathrm{HTT}$. $\mathrm{BP}_{\mathrm{ND}}$ was thresholded until only activity in area of raphé nuclei was visible.

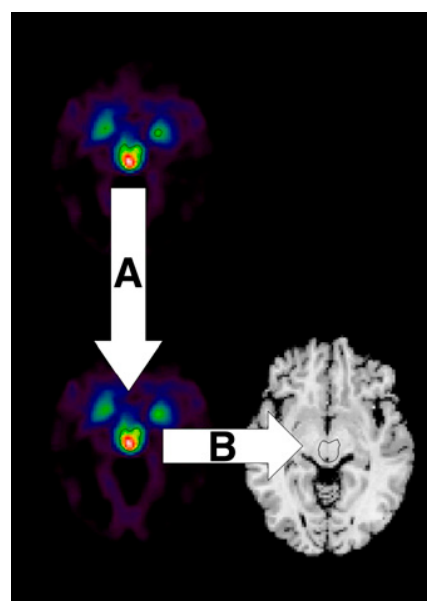

FIGURE 2. Creation of template: delineation of rostral raphé nuclei on parametric PET image by increasing threshold until no activity outside raphé area is included $(A)$ and then transferring VOI to coregistered MR image (B).

These templates are then used as described by Svarer et al. (10) to create a probabilistic map of the raphé region for new subjects, which is threshold-independent of the binding potential images for these subjects.

\section{Creating the Template Set}

The raphé nuclei VOIs on the parametric images (representing $\mathrm{BP}_{\mathrm{ND}}$ for each voxel) were identified for the 10 template subjects by applying an individually determined threshold. First, the threshold at the level of the rostral midbrain or the interpeduncular fossa, identified on the coregistered MR image, was established; this threshold was then fixed on consecutive slices in the caudal direction. The threshold was settled on the first slices in a strictly reproducible manner by gradually increasing the threshold of $\mathrm{BP}_{\mathrm{ND}}$ (using 0.01 steps) until at no level did the VOI exceed the mediolateral brain stem (Fig. 2). According to histologic (1) and cytoarchitectonic (13) studies, it is at this level that we expected raphé activity. Usually, the

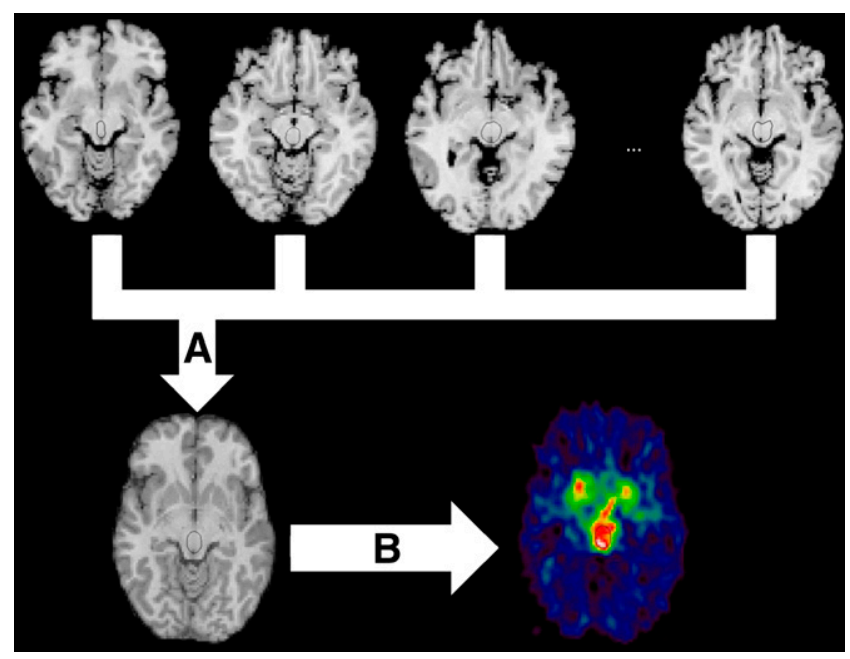

FIGURE 3. Warping from several template MR images $(n=$ 10) onto MR image of test subject creates probabilistic VOI (A); in second step, $\mathrm{VOI}$ is transferred from MR image of test subject to coregistered PET image (B). 
last remaining activity outside the raphé was small nonconfluent volumes in the vicinity of thalamus.

In this way, for all 10 images, the VOI encompassed the rostral portion of the raphé nuclei, mainly the nucleus raphé dorsalis. The VOI was then transferred to each subject's coregistered MR image to be used for the subsequent warping procedure (Fig. 3). Additionally, we used our common template set as described earlier (10) to create a probabilistic VOI for the cerebellum, thalamus, caudate, and putamen to determine $k_{2}{ }^{\prime}$ from the extracted time-activity curves for reference binding (cerebellum) and high-binding regions (thalamus, caudate, and putamen).

\section{Using Template Set to Create Observer-Independent Probabilistic VOI}

In a method similar to the one employed by Svarer et al. (10), we then used the resulting $10 \mathrm{VOI}$ templates to define the raphé nuclei region for the remaining 10 test subjects (Fig. 3). In short, the probabilistic VOIs are created by the estimation of a warp field from each of the 10 template MR images to the MR image of the individual, and the warp field is then applied to the template raphe nuclei VOI. In each new subject, the 10 warped VOIs constitute a unique probabilistic map that is thresholded to define the final VOI. This threshold is automatically determined by adjusting it so the volume of the generated region has the same volume as the mean regional volume of all the transferred template regions, as described in Svarer et al. (10). Accordingly, the generated VOI had a volume that fit the volume of the brain region in the new subject and, thereby, the brain size for that subject.

\section{Comparison of Probabilistic and Manual Delineation}

For comparison, manual delineation was also performed, as described by other groups $(2,7)$. Starting at the level in which the interpeduncular fossa was first clearly visible and continuing in the caudal direction, circles with a fixed diameter $(6 \mathrm{~mm})$ were drawn directly on 4-5 slices on the summed PET images centered around the highest activity. This task was performed independently for the same 10 test subjects by 3 trained observers.

To evaluate the effect of the VOI determined by either approach (probabilistic or manual), time-activity curves were extracted for all 10 test subjects and the raphé nuclei $\mathrm{BP}_{\mathrm{ND}}$ was calculated for each subject as mentioned above. We used a 2-way ANOVA to compare $\mathrm{BP}_{\mathrm{ND}}$ and $\chi^{2}$ between the automatic and the manual approaches. Additionally, for evaluation of the interobserver variability, we calculated the interclass correlation coefficient between all 3 observers using Matlab-based software (MathWorks).

\section{RESULTS}

The probabilistic map approach resulted in a volume for the raphé VOI that was more than twice as large as the VOI derived from the manual approach $(1.41 \pm 0.12 \mathrm{~mL}$ vs. $0.58 \pm$ $0.04 \mathrm{~mL}$ ). The interclass correlation coefficient for the observer-dependent delineation approach was only 0.47 . Time-activity curves derived from the observer-independent approach showed less noise, resulting in a better fit represented by a significantly lower $\chi^{2}$ value (Fig. 4B), compared with the value determined from manual delineation by 3 different observers. Values were $12.57 \pm 4.29$ (probabilistic VOI), $15.30 \pm 5.41$ (observer 1), $14.51 \pm 4.72$ (observer 2), and $14.50 \pm 5.48$ (observer 3 ). Probabilistic VOI delineation, compared with manual delineation, resulted in a significantly lower $\chi^{2}$ value $(P<0.001 ; 2$-way ANOVA). As shown in Figure $4 \mathrm{~A}, \mathrm{BP}_{\mathrm{ND}}$ resulting from the probabilistic map approach, compared with manual delineation, was $4.40 \pm$ 0.44 (probabilistic VOI), $3.65 \pm 0.65$ (observer 1), $3.58 \pm$ 0.62 (observer 2), and $3.45 \pm 0.57$ (observer 3 ); thus, probabilistic VOI delineation, compared with manual delineation, resulted in a significantly higher $\mathrm{BP}_{\mathrm{ND}}$ value $(P<$ $0.001 ; 2$-way ANOVA).

\section{DISCUSSION}

With an extension of the probability map-based approach, we have shown here the feasibility for objective identification of functional brain VOIs that are not identified by their anatomic structure but rather by their anatomic relationship to other brain structures. The templates were created on the basis of the activity contrast to the neighboring region, the thalamus. Although this approach may seem to be somewhat arbitrary, the resulting volumes were consistently defined and served the purpose of raphé delineation in the independent sample. Further, although the method returns volumes that are more than 2 times larger than those found by a manual approach, the resulting $\mathrm{BP}_{\mathrm{ND}}$ was significantly higher and goodness of fit was improved, supporting the idea that the raphé VOI is more correctly identified. In accordance with the improved goodness of fit, we observed less noisy timeactivity curves for the raphé (probably largely due to the

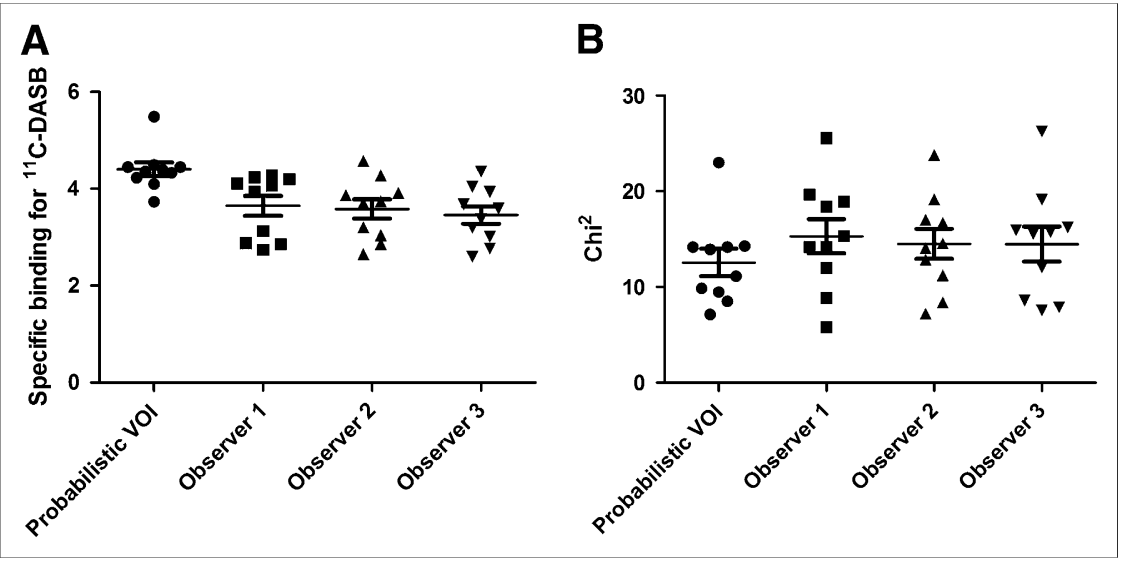

FIGURE 4. $\quad \mathrm{BP}_{\mathrm{ND}}(\mathrm{A})$ and resulting $\chi^{2}$ (B) obtained using probabilistic $\mathrm{VOI}$ and VOI of 3 observers. Horizontal lines represent means, and bars indicate SD. 
inclusion of a larger volume) associated with better count statistics. The volume of the probabilistic VOI $(\sim 1.4 \mathrm{~mL})$ exceeds what was found in cytoarchitectonic studies in humans for the rostral raphé nuclei $(\sim 70 \mathrm{~mL})(13)$. This is mainly caused by 2 factors. First, the probabilistic VOI we created merged activity of all subnuclei of the nucleus raphé dorsalis in 1 region, including most of the interfascicular, ventral, ventrolateral, and dorsal portions. The manually delineated VOI usually did not include activity from the interfascicular and ventrolateral subnuclei. Second, the probabilistic VOI is based on and created for activity measured with PET, which suffers from low resolution and partial-volume effects and is, thus, less precise than activity measured using histologic sections. On the other hand, the raphé region is difficult to accurately outline in histologic sections, which further may have undergone some shrinkage. We do not attempt to validate the appropriateness of the identified raphé volume. However, the probabilistic VOI approach is far superior to the current alternative, namely manual delineation on the functional image, which is labor-intensive, subjective, less reproducible, and potentially biased in disorders in which the raphé activity is altered.

The large interobserver variability observed with the manual approaches was surprising, particularly because the observers are experts in anatomic delineations and they received the same instructions. Our finding suggests that results published about manual approach-defined raphé regions are likely to be associated with considerable noise, not only because of the more noisy time-activity curves but also because of the larger interobserver variability and smaller $\mathrm{BP}_{\mathrm{ND}}$, which requires larger sample sizes to correctly identify group differences or associations. To build the probability map, we used templates from 10 healthy subjects and we did not attempt to validate the effect of including more or fewer subjects. We have, however, previously shown that inclusion of 6 subjects is enough to stably identify even small VOIs correctly (10) and found that inclusion of additional subjects or templates did not significantly improve the estimates. An alternative approach would be to create a probabilistic map in standard space, and that could easily have been done. It has previously been shown, however, that this approach is inferior to a probabilistic map created on the basis of 10 independent templates $(10,14)$.

The defined VOI encompassed the rostral portion of the raphé nuclei only, mainly the nucleus raphé dorsalis (Fig. 1). Although the fact that only the rostral portion is included may be seen as a relative limitation, the rostral group of the raphé nuclei produces more than $85 \%$ of serotonin in the entire human brain. However, the approach would need to be properly validated for assessment of, for example, $5-\mathrm{HT}_{1 \mathrm{~A}}$ receptor binding.

Although in this particular case we showed the approach to be valid for 5-HTT binding in the raphé nuclei region, the approach is principally applicable for any functional brain region, no matter which imaging modality (e.g., SPECT, PET, and functional MRI [fMRI]) is used, as long as the functional image comes with a structural image of sufficient quality. Because of the large intersubject variability of the structure-function relationship in fMRI studies, it may be more difficult to create VOIs based on information obtained with this technique, compared with receptor PET. However, functional delineation of the brain by neurotransmitter receptor fingerprints as described by Zilles et al. (15) would be an obvious way to apply the probability mapbased approach, by combining different templates for different tracers to ultimately create a probabilistic map of the receptor fingerprint of a functional region.

\section{CONCLUSION}

We have presented a fast, observer-independent probability map-based method that yields less noisy data and higher binding potentials and is more reproducible for quantification of the serotonin transporter binding in the raphé nuclei. The approach may prove useful for delineations of other functional brain regions that lack a clear anatomic correlate, in which functionality is defined with molecular imaging, fMRI, or magnetoencephalography.

Templates and software for the probabilistic VOI map are freely available and included in the software package for partial-volume correction (PVElab; http://nru.dk/pveout).

\section{ACKNOWLEDGMENTS}

This study was funded by the Lundbeck Foundation and by the EU 6th Framework program DiMI (LSHB-CT-2005512146). The John and Birthe Meyer Foundation is gratefully acknowledged for the donation of the cyclotron and PET scanner.

\section{REFERENCES}

1. Hornung JP. The human raphé nuclei and the serotonergic system. J Chem Neuroanat. 2003;26:331-343.

2. Drevets WC, Frank E, Price JC, et al. PET imaging of serotonin 1A receptor binding in depression. Biol Psychiatry. 1999;46:1375-1387.

3. Becker G, Becker T, Struck M, et al. Reduced echogenicity of brainstem raphé specific to unipolar depression: a transcranial color-coded real-time sonography study. Biol Psychiatry. 1995;38:180-184.

4. Cannon D, Ichise M, Rollis D, et al. Elevated serotonin transporter binding in major depressive disorder assessed using positron emission tomography and $\left[{ }^{11}\right.$ C $]$ DASB; comparison with bipolar disorder. Biol Psychiatry. 2007;62:870877.

5. Cannon DM, Ichise M, Fromm SJ, et al. Serotonin transporter binding in bipolar disorder assessed using $\left[{ }^{11} \mathrm{C}\right] \mathrm{DASB}$ and positron emission tomography. Biol Psychiatry. 2006;60:207-217.

6. Rub U, Del Tredici K, Schultz C, Thal DR, Braak E, Braak H. The evolution of Alzheimer's disease-related cytoskeletal pathology in the human raphé nuclei. Neuropathol Appl Neurobiol. 2000;26:553-567.

7. Lundberg J, Odano I, Olsson H, Halldin C, Farde L. Quantification of ${ }^{11} \mathrm{C}$ MADAM binding to the serotonin transporter in the human brain. $\mathrm{J} \mathrm{Nucl} \mathrm{Med}$. 2005;46:1505-1515.

8. Jovanovic H, Cerin A, Karlsson P, Lundberg J, Halldin C, Nordstrom AL. A PET study of 5-HT1A receptors at different phases of the menstrual cycle in women with premenstrual dysphoria. Psychiatry Res. 2006;148:185-193.

9. Borg J, Andree B, Soderstrom H, Farde L. The serotonin system and spiritual experiences. Am J Psychiatry. 2003;160:1965-1969.

10. Svarer C, Madsen K, Hasselbalch SG, et al. MR-based automatic delineation of volumes of interest in human brain PET images using probability maps. Neuroimage. 2005;24:969-979. 
11. Houle S, Ginovart N, Hussey D, Meyer JH, Wilson AA. Imaging the serotonin transporter with positron emission tomography: initial human studies with $\left[{ }^{11} \mathrm{C}\right] \mathrm{DAPP}$ and $\left[{ }^{11} \mathrm{C}\right] \mathrm{DASB}$. Eur J Nucl Med. 2000;27:17191722.

12. Ichise M, Liow JS, Lu JQ, et al. Linearized reference tissue parametric imaging methods: application to $\left[{ }^{11} \mathrm{C}\right] \mathrm{DASB}$ positron emission tomography studies of the serotonin transporter in human brain. J Cereb Blood Flow Metab. 2003;23:10961112
13. Baker KG, Halliday GM, Tork I. Cytoarchitecture of the human dorsal raphé nucleus. J Comp Neurol. 1990;301:147-161.

14. Heckemann RA, Hajnal JV, Aljabar P, Rueckert D, Hammers A. Automatic anatomical brain MRI segmentation combining label propagation and decision fusion. Neuroimage. 2006;33:115-126.

15. Zilles K, Palomero-Gallagher N, Grefkes C, et al. Architectonics of the human cerebral cortex and transmitter receptor fingerprints: reconciling functional neuroanatomy and neurochemistry. Eur Neuropsychopharmacol. 2002;12:587-599. 